\title{
Estimation of sea-ice physical parameters using polarimetric SAR: results from Okhotsk and Lake Saroma campaign
}

\author{
Hiroyuki Wakabayashi, ${ }^{1}$ Takeshi Matsuoka, ${ }^{2}$ Kazuki Nakamura, ${ }^{3}$ Fumihiko Nishio ${ }^{3}$ \\ ${ }^{1}$ Earth Observation Research Center (EORC), National Space Development Agency of Japan (NASDA), 1-9-9 Roppongi, \\ Minato-ku, Tokyo 106-0032, Japan \\ ${ }^{2}$ Communications Research Laboratory, 4-2-1 Nukuikita, Koganei, Tokyo 184-8795, Japan \\ ${ }^{3}$ Center for Environmental Remote Sensing, Chiba University, 1-33 Yaoicho, Inage-ku, Chiba 263-8522, Japan
}

\begin{abstract}
We have acquired ground-truth data at Lake Saroma, northeast Hokkaido, Japan, and the surrounding area since 1993 in order to collect data on regional sea ice in the Sea of Okhotsk. The data were acquired in 1999 by polarimetric and interferometric SAR (Pi-SAR), the dual-frequency, fully polarimetric airborne SAR system jointly developed by the National Space Development Agency of Japan (NASDA) and the Communications Research Laboratory (CRL), simultaneously with ground experiments. This paper describes the results of polarimetric data analysis of typical sea ice observed in the offshore region near Lake Saroma. The polarimetric parameters used were correlation coefficient and phase difference. Based on the analysis of these parameters, we found that the correlation coefficient between RR and LL polarizations can discriminate four categories including three types of ice and open water.
\end{abstract}

\section{INTRODUGTION}

The Sea of Okhotsk is located in the most southerly region of the Northern Hemisphere in which sea ice exists during winter. Since the area and volume of sea ice in this region are related to climate change locally as well as globally (Nishio and Aota, 1993), it is important to develop methods for monitoring sea-ice parameters around this region. Currently, we are analyzing sea-ice characteristics and developing monitoring algorithms using synthetic aperture radar (SAR) on board the Japanese Earth Resources Satellite (JERS-1), European Remote-sensing Satellites 1 and 2 (ERS-1/-2) (Wakabayashi and Nishio, 1996) and RADARSAT. We plan to apply the developed algorithms on a routine basis in the future. We are also preparing to use fully polarimetric SAR data from future space-borne SARs, such as RADARSAT-2 and the Advanced Land Observing Satellite (ALOS).

In order to gather data for sea-ice monitoring in the Sea of Okhotsk, we have acquired ground-truth data at Lake Saroma, northeast Hokkaido, Japan, and its surrounding area since 1993. The polarimetric and interferometric SAR (Pi-SAR), the dual-frequency, fully polarimetric airborne SAR system developed by the National Space Development Agency of Japan (NASDA) and the Communications Research Laboratory (CRL), acquired the data in 1999 simultaneously with ground experiments. NASDA plans to use the Pi-SAR L-band system to calibrate and validate future space-borne SAR systems, such as the Phased Array-type L-band SAR (PALSAR) on board ALOS.

Polarimetric SAR changes the polarization of its transmitted signal pulse-by-pulse to either linear horizontal or linear vertical, and then receives two differently polarized signals simultaneously. Therefore, it can acquire four polarimetric combinations, $\mathrm{HH}, \mathrm{VV}, \mathrm{HV}$ and $\mathrm{VH}$. Polarimetric SAR data are generally considered to contain much more information on scattering characteristics of the targets than the single-polarization SAR data. For example, the copolarization dataset, $\mathrm{HH}$ and $\mathrm{VV}$, has surface scattering information, and the cross-polarization dataset, $\mathrm{HV}$ and $\mathrm{VH}$, has volume-scattering information.

This paper describes the preliminary results of polarimetric data analysis of typical sea ice observed in the offshore region near Lake Saroma.

\section{TEST SITE}

Figure 1 shows our test site including Lake Saroma and its surrounding area observed by Pi-SAR and RADARSATon 23 February 1999. RADARSAT acquired the data in an ascending orbit. Pi-SAR was operated simultaneously with RADARSAT data acquisition. Lake Saroma was fully covered by ice with a relatively smooth surface. We use Lake Saroma as the ground-truth site because it is a salt-water lake connected to the Sea of Okhotsk through two channels, so the covering ice has almost the same salinity as sea ice in the open sea. We measured ice thickness, bulk salinity and surface roughness at 25 points on the lake. We also have several ice-thickness data measured from ship at the offshore region near Abashiri. This paper focuses on sea ice in the southern Sea of Okhotsk in mid-February 1999. Using the ground-truth data we have acquired on Lake Saroma and its surrounding area during the past several years, we found that the snow on the ice is dry and that the mean salinity of the snow-ice interface was around 8 ppt. The maximum snow depth is $15 \mathrm{~cm}$ and the ice thickness is $70 \mathrm{~cm}$ (Wakabayashi and Nishio, 1996). 


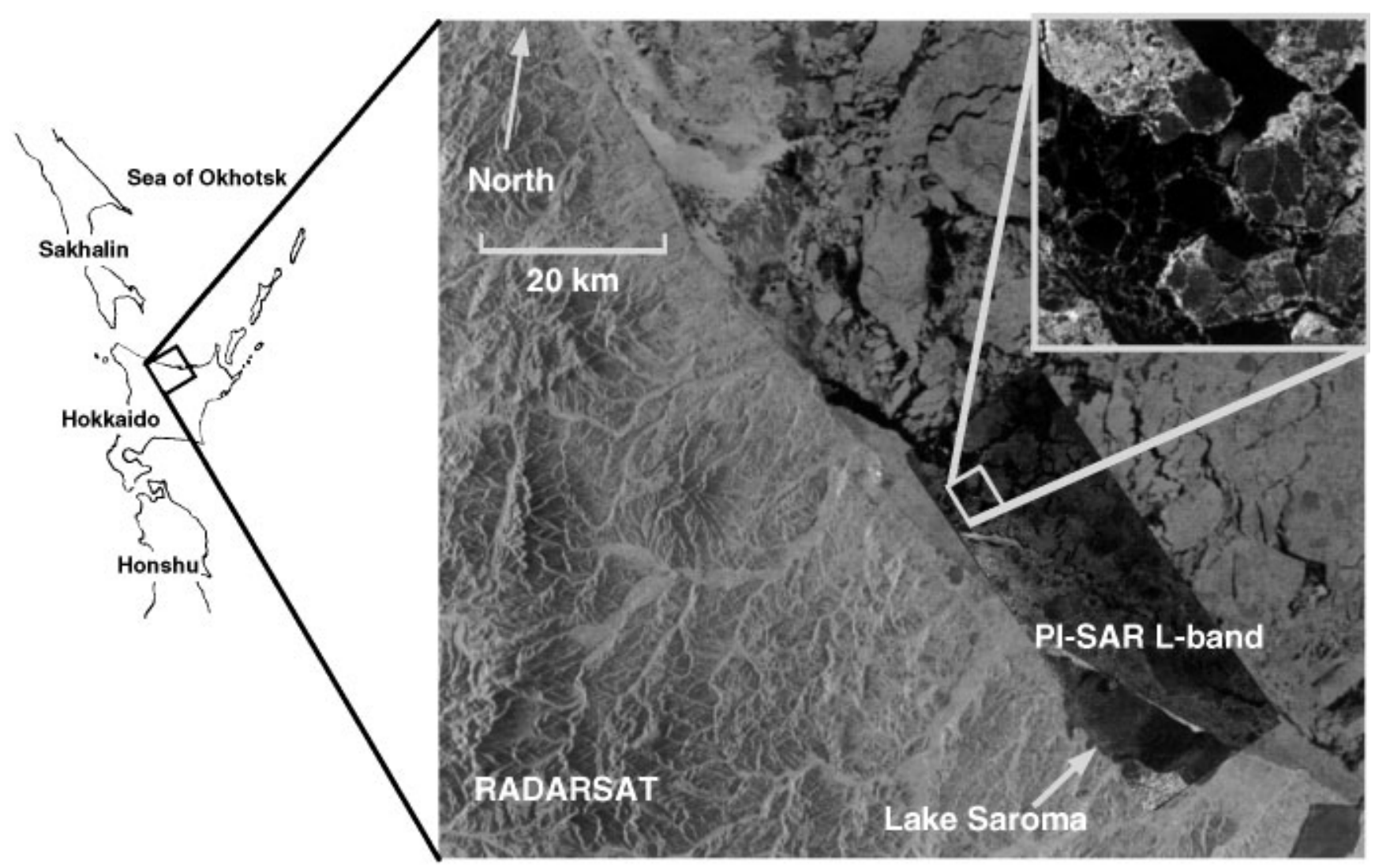

Fig. 1. Example of images acquired by RADARSAT and Pi-SAR over the test site.

\section{Pi-SAR L-BAND SYSTEM}

\subsection{L-band system characteristics}

The basic characteristics of the Pi-SAR L-band system are compared with those of the PALSAR system in Table 1 . In order to use this system for calibrating and validating PALSAR, the center frequency and range of observing incidence angles were taken to be almost the same as for the PALSAR system. However, radiometric and geometric characteristics, such as noise-equivalent backscattering coefficient and spatial resolution, were designed to outperform the PALSAR system.

\subsection{Calibration procedure}

Several calibration methods have been proposed for existing polarimetric SAR data, such as the Jet Propulsion Laboratory's (JPL, Pasadena, CA, U.S.A.) AIRSAR and SIR-C data (Freeman and others, 1990; Van Zyl, 1990; Sarabandi and others, 1992). In general, cross-talk between the $\mathrm{H}$ and $\mathrm{V}$ channels is a function of incidence angle. When methods to estimate cross-talk parameters from the images of man-made targets, such as a transponder or a corner reflector, are applied, we need to deploy many reflectors along the crosstrack line to cover the whole range of incidence angles. This could be difficult if only a small calibration site is available. In addition, our Pi-SAR L-band system is considered to be a reciprocal system. Therefore, we use a natural target to estimate cross-talk parameters (Van Zyl, 1990).

Taking $Z$ as an observed scattering matrix and $S$ as a scattering matrix of the target, the relation between the two matrices is as follows:

$$
\begin{aligned}
& Z=A \mathrm{e}^{j \phi} R S T+N \\
& {\left[\begin{array}{cc}
z_{\mathrm{hh}} & z_{\mathrm{hv}} \\
z_{\mathrm{vh}} & z_{\mathrm{vv}}
\end{array}\right]=A \mathrm{e}^{j \phi}\left[\begin{array}{cc}
1 & \delta_{2} \\
\delta_{1} & f
\end{array}\right]\left[\begin{array}{ll}
s_{\mathrm{hh}} & s_{\mathrm{hv}} \\
s_{\mathrm{vh}} & s_{\mathrm{vv}}
\end{array}\right] } \\
& \cdot\left[\begin{array}{cc}
1 & \delta_{1} \\
\delta_{2} & f
\end{array}\right]+\left[\begin{array}{ll}
n_{\mathrm{hh}} & n_{\mathrm{hv}} \\
n_{\mathrm{vh}} & n_{\mathrm{vv}}
\end{array}\right],
\end{aligned}
$$

where $A$ is an absolute calibration coefficient, $\phi$ is the phase related to the propagation distance between SAR and the target, $R$ and $T$ are matrices that represent the radar polarimetric characteristics for receive and transmit, $\delta_{1}$ and $\delta_{2}$ are cross-talk parameters, $f$ is the gain imbalance between $\mathrm{HH}$ and VV channels, and $N$ is the noise matrix in each channel.

Table 1. Pi-SAR L-band system characteristics compared with satellite-borne PALSAR system

\begin{tabular}{lcc}
\hline & Pi-SAR/L-SAR & ALOS $/$ PALSAR \\
\hline Center frequency & $1.27 \mathrm{GHz}$ & $1.27 \mathrm{GHz}$ \\
Transmission peak power & $3.5 \mathrm{~kW}$ & $2.4 \mathrm{~kW}$ \\
Chirp bandwidth & $50 \mathrm{MHz}$ & $28 \mathrm{MHz} / 14 \mathrm{MHz}$ \\
Antenna size & $1.6 \mathrm{~m} \times 0.7 \mathrm{~m}$ & $8.9 \mathrm{~m} \times 3.5 \mathrm{~m}$ \\
Polarization & $\mathrm{HH} / \mathrm{HV} / \mathrm{VH} / \mathrm{VV}$ & $\mathrm{HH} / \mathrm{HV} / \mathrm{VH} / \mathrm{VV}$ \\
Incidence angle & $20-60^{\circ}(\mathrm{fixed})$ & $8-60^{\circ}(\mathrm{variable})$ \\
Observation swath & $42.5 \mathrm{~km} / 19.6 \mathrm{~km}$ & $70 \mathrm{~km}$ \\
Spatial resolution & $3 \mathrm{~m}(4 \mathrm{look})$ & $10 \mathrm{~m}(2 \mathrm{look}) 20 \mathrm{~m}(4 \mathrm{look})$ \\
Bit length & $8 \mathrm{bits}(1 \mathrm{and} \mathrm{Q}$ & $5 \mathrm{bits}(1 \mathrm{and} \mathrm{Q})$ \\
Noise-equivalent & $<-35 \mathrm{~dB}$ & $-25 \mathrm{~dB}$ \\
\multicolumn{1}{c}{ backscattering coefficient } & & \\
& &
\end{tabular}




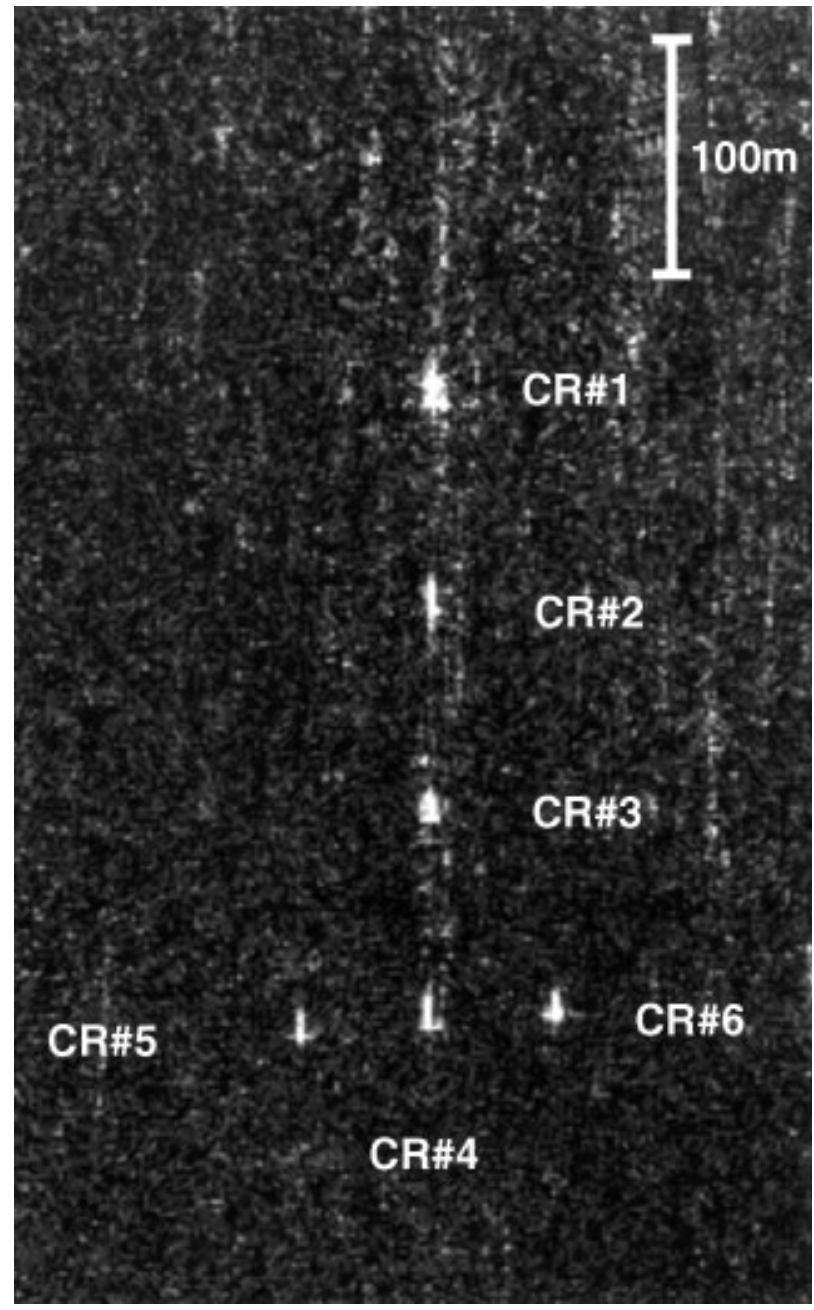

Fig. 2. Example of corner reflectors deployed on Lake Saroma.
When the noise term in Equation (1) is neglected, the scattering matrix of the observed target is derived as follows:

$$
S=\frac{1}{A \mathrm{e}^{j \phi}} R^{-1} Z T^{-1} .
$$

The following is the procedure we adopted before the data analysis.

(a) Cross-talk parameters are determined with an iterative method using data from natural targets having no correlation between like and cross-channels.

(b) The gain imbalance is estimated by observing the trihedral corner reflectors.

(c) The absolute calibration coefficient is derived from the theoretical cross-sections of the corner reflectors.

The natural targets that satisfy condition (a) are considered to be an azimuthally symmetric target for which the dominant scatterer is surface scattering. We believe that salty first-year sea ice satisfies the above condition, because it has relatively high salinity and its surface roughness has no azimuth orientation dependency.

\subsection{Calibration results}

Since the backscattering coefficient of smooth, salt-water ice is considered to be small, a high signal-to-clutter ratio for reflectors could be expected. In this experiment, we deployed six corner reflectors on the ice cover in Lake Saroma. The rectangular trihedral corner reflectors with leg lengths of 60 and $85 \mathrm{~cm}$ were used to estimate the gain imbalance and absolute calibration coefficient, and $80 \mathrm{~cm}$ dihedral corner reflectors were used to check polarization signatures. Figure 2 presents a zoomed image around the area where we deployed the corner reflectors.

The procedure described in the previous section was applied to the image over Lake Saroma and the target seaice area. Cross-talk was estimated by using the homogeneous area in each image, and the gain imbalance was estimated at

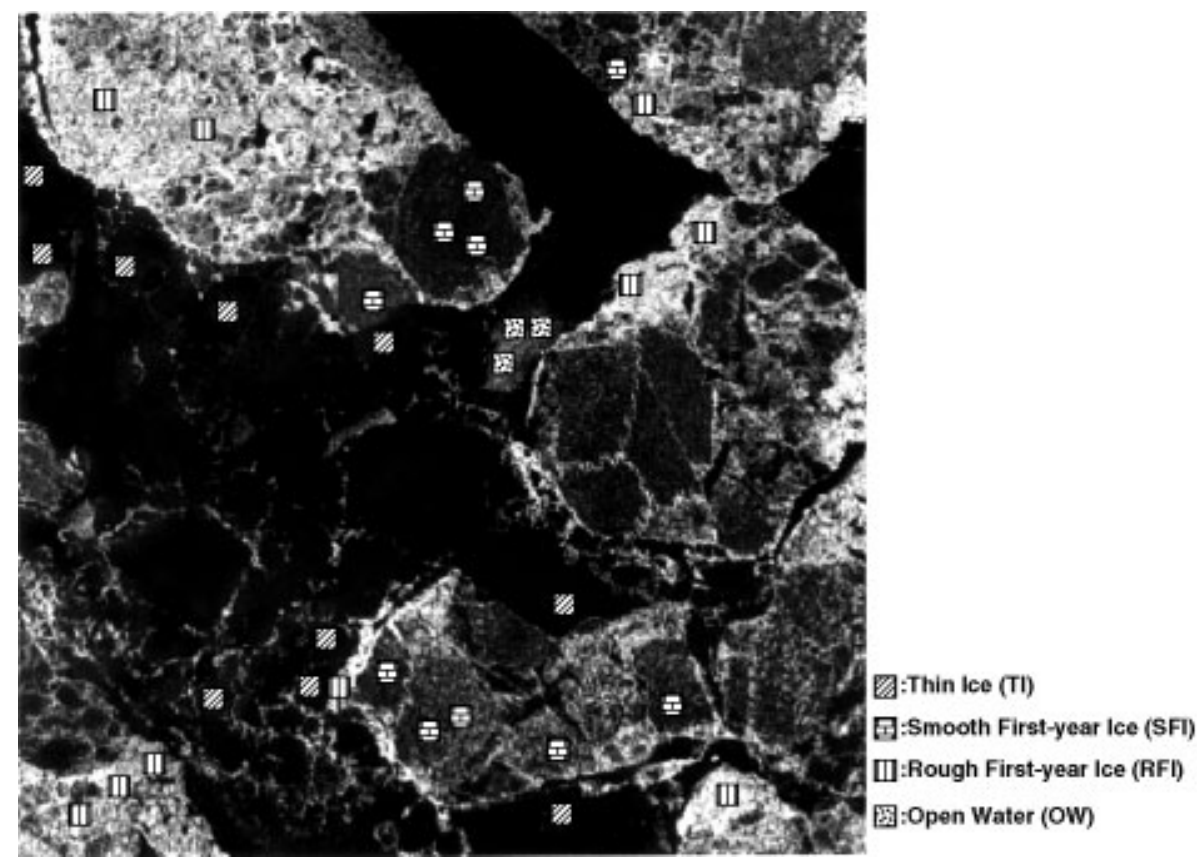

Fig. 3. Evaluated points of four categories in the area of interest are plotted on the Pi-SAR L-band total power image. The size of this area is $4 \mathrm{~km}$ by $4 \mathrm{~km}$. 

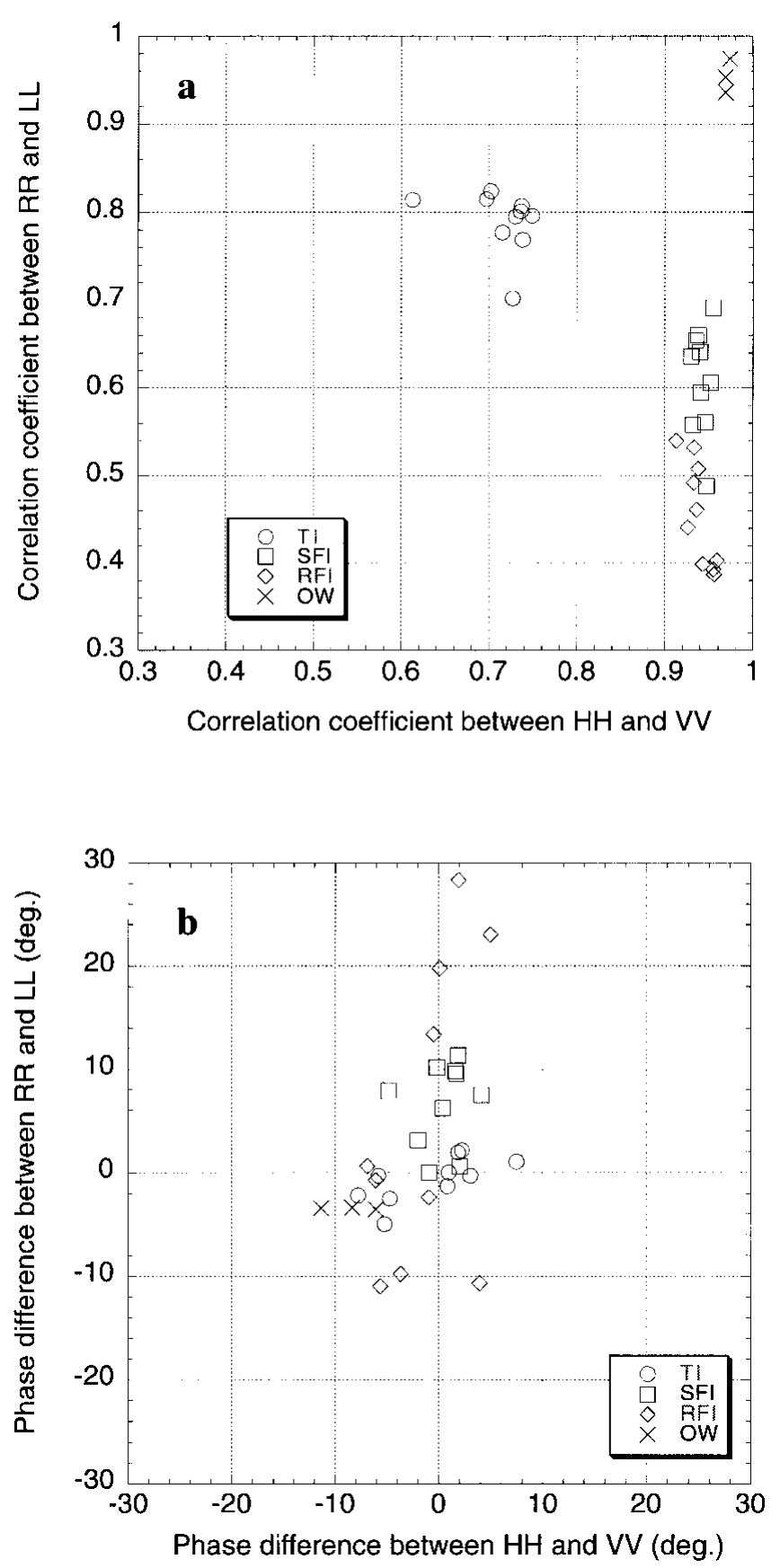

Fig. 4. Polarimetric characteristics of typical ice. (a) Correlation coefficient; (b) phase difference.

the peak of each trihedral reflector. The values from four trihedral reflectors were averaged. After calculating the target scattering matrix by substituting the estimated parameters into Equation (2), we calculated an absolute calibration coefficient using the integration method (Gray and others, 1990). The accuracy of the estimated calibration factor is within $1.1 \mathrm{~dB}$ for all reflectors (Wakabayashi and others, 1999).

\section{POLARIMETRIC DATA ANALYSIS OF THE SEA ICE}

Rignot and Drinkwater (1994) analyzed L- and C-band polarimetric data acquired by JPL AIRSAR covering the Beaufort Sea in 1988 and found that the dual-frequency single-polarization data classify sea ice more accurately than the single-frequency dual-polarization data. Winebrenner and others (1995) used the same dataset and found that the co-polarization ratio and phase were related to ice thickness. Nghiem and others (1995a, b) created a polarimetric signature model for sea ice and tried to explain the backscattering coefficients from multi-year ice, first-year ice and thin ice in leads in the Beaufort Sea. As an example of SIR-C L-band polarimetric data, Eriksson and others (1998) analyzed the first-year ice in the eastern Weddell Sea, Antarctica, and found that the correlation coefficient between $\mathrm{HH}$ and $\mathrm{VV}$ combined with VV backscattering coefficient could achieve higher classification accuracy.

Barber and Nghiem (1999) reported that the thermodynamic structure of the snow-ice boundary, relating to the dielectric-constant changes in the snow basal layer and ice surface layer, affected the C-band backscattering coefficient during the melting period. However, since we acquired Lband data in February, we believed that the dominant scattering mechanism of the winter sea ice in this region was the ice surface scattering (Wakabayashi and Nishio, 1996).

Sea ice in the area of interest shown in Figure 1 was classified into four categories based on the backscattering coefficient in C-band of RADARSAT and L- and X-bands of Pi-SAR data (Matsuoka and others, in press). The four categories were thin ice (TI), smooth first-year ice (SFI), rough first-year ice (RFI) and open water (OW). In Figure 3, rectangles indicate the sampling points from the four categories. Using the results of the previous work, we focused on analyzing the correlation coefficient and the phase difference between $\mathrm{HH}$ and VV. We also analyzed the correlation coefficient and the phase difference between RR and LL, which were calculated from the polarization synthesis and contained cross-polarization information.

Although the previous work indicated that the co-polarization ratio yielded information related to ice thickness, we could not get any valuable results in this time, probably because the data analyzed were acquired at a relatively small incidence angle.

By taking $\rho_{\text {ppqq }}$ and $\phi_{\text {ppqq }}$ as the correlation coefficient and phase difference between pp and qq polarizations, these values are calculated from the scattering matrix as follows:

$$
\rho_{\text {ppqq }} \exp \left(j \phi_{\text {ppqq }}\right)=\frac{\left\langle S_{\mathrm{pp}} S_{\mathrm{qq}}^{*}\right\rangle}{\sqrt{\left\langle S_{\mathrm{pp}} S_{\mathrm{pp}}^{*}\right\rangle\left\langle S_{\mathrm{qq}} S_{\mathrm{qq}}^{*}\right\rangle}},
$$

where $S_{\mathrm{pp}}$ and $S_{\mathrm{qq}}$ are the elements of the scattering matrix, and \langle\rangle indicates the ensemble average.

$\rho_{\mathrm{HHVV}}$ is the correlation coefficient between the $\mathrm{HH}$ and VVchannels. In general, $\rho_{\mathrm{HHVV}}$ approaches 1 if the dominant scatter is surface scattering. $\phi_{\mathrm{HHVV}}$ is the phase difference between $\mathrm{HH}$ and VV channels, and approaches 0 for surface scattering. We also analyzed $\rho_{\text {RRLL }}$ and $\phi_{\text {RRLL, which are }}$ calculated by polarimetric synthesis.

Figure 4 shows cross-plots of correlation coefficients and phase differences from the sampling points in Figure 3. From these plots we can conclude the following:

(1) The difference of $\rho_{\mathrm{HHVV}}$ between SFI and RFI is very small. $\rho_{\mathrm{HHVV}}$ of TI has smaller values than for the other three categories.

(2) $\rho_{\text {RRLL }}$ decreases in the order TI, SFI and RFI. We can separate three ice types using $\rho_{\text {RRLL }}$.

(3) $\phi_{\mathrm{HHVV}}$ and $\phi_{\text {RRLL }}$ provide less information regarding ice types and discrimination between ice and water.

We also analyzed the incidence-angle dependency on correlation coefficients and phase differences. Although the 
incidence angle in Figure 3 ranged from $21^{\circ}$ to $36^{\circ}$, little angle dependency was found.

It was reported that $\rho_{\text {RRLL }}$ was sensitive to surface roughness of the soil and could be used to retrieve roughness parameters for pre-processing the soil-moisture estimation (Mattia and others, 1997). Since the surface roughnesses of three ice types seem to have different characteristics, we may conclude that $\rho_{\text {RRLL }}$ is a good indicator for describing surface roughness of salty first-year ice.

\section{CONGLUSION}

By using L-band SAR data acquired by Pi-SAR, we performed polarimetric analysis of sea ice observed in the Sea of Okhotsk. The polarimetric parameters used were correlation coefficient and phase difference. Based on the analysis of these parameters, we found that the correlation coefficient between RR and LL polarizations enables us to discriminate four categories including three types of ice and open water.

In order to derive a method to estimate surface roughness parameters of sea ice, it is necessary to compare $\rho_{\mathrm{RRLL}}$ with the measured surface roughness as a next step.

\section{AGKNOWLEDGEMENTS}

The authors are grateful to the students of Hokkaido University of Education for their help in acquiring the groundtruth data as well as deploying corner reflectors.

\section{REFERENGES}

Barber, D. G. and S.V. Nghiem. 1999. The role of snow on the thermal dependence of microwave backscatter over sea ice. 7. Geophys. Res., 104(C11), 25,789-25,803.

Eriksson, L., M. R. Drinkwater, B. Holt, E. Valjavek and O. Nortier. 1998. SIR-C polarimetric radar results from the Weddell Sea, Antarctica. In IGARSS '98. 18th International Geoscience and Remote Sensing Symposium, 6-10
Fuly 1998, Seattle, Washington. Proceedings. Vol.4. Piscataway, NJ, Institute of Electrical and Electronics Engineers, 2222-2224.

Freeman, A., Y. Shen and C.W. Werner. 1990. Polarimetric SAR calibration experiment using active radar calibrators. IEEE Trans. Geosci. Remote Sensing, GE-28(2), 224-40.

Gray, A. L., P. W. Vachon, C. E. Livingstone and T. I. Lukowski. 1990. Synthetic aperture radar calibration using reference reflectors. IEEE Trans. Geosci. Remote Sensing, GE-28(3), 374-383.

Matsuoka, T. and 8 others. In press. Multifrequency polarimetric airborne SAR observations of sea ice in the Sea of Okhotsk. In 13th International Symposium on Sea Ice and the Sea of Okhotsk, 5-9 February 2000, Mombetsu, Japan. Proceedings. Mombetsu, Japan, Okhotsk Sea and Cold Ocean Research Association.

Mattia, F. and 6 others. 1997. The effect of surface roughness on multifrequency polarimetric SAR data. IEEE Trans. Geosci. Remote Sensing, GE-35(4), 954-966.

Nghiem, S.V., R. Kwok, S. H. Yueh and M. R. Drinkwater. 1995a. Polarimetric signatures of sea ice. Part I: Theoretical model. F. Geophys. Res., $\mathbf{1 0 0}(\mathrm{C} 7), 13,665-13,679$.

Nghiem, S.V., R. Kwok, S. H. Yueh and M. R. Drinkwater. 1995b. Polarimetric signatures of sea ice. Part II: Experimental observations. F. Geophys. Res., 100 (C7), 13,681-13,698.

Nishio, F. and M. Aota. 1993. Variability of sea ice extent in the Okhotsk Sea. In 8th International Symposium on Sea Ice and the Sea of Okhotsk, 1-5 February 1993, Mombetsu, Japan. Proceedings. Mombetsu, Japan, Okhotsk Sea and Cold Ocean Research Association, 305-307.

Rignot, E. and M. R. Drinkwater. 1994. Winter sea-ice mapping from multiparameter synthetic-aperture radar data. f. Glaciol., 40(134), 31-45.

Sarabandi, K., L. E. Pierce and F.T. Ulaby. 1992. Calibration of polarimetric imaging SAR. IEEE Trans. Geosci. Remote Sensing, GE-30(3), 540-549.

Van Zyl, J. J. 1990. Calibration of polarimetric radar images using only images parameters and trihedral corner reflector responses. IEEE Trans. Geosci. Remote Sensing, GE-28(3), 337-348.

Wakabayashi, H. and F. Nishio. 1996. A study of ice on Lake Saroma using SAR data. 7. Remote Sensing Soc. Jpn, 16 (2), 59-66.

Wakabayashi, H., T. Kobayashi, M. Satake and S. Uratsuka. 1999. Airborne L-band SAR system: characteristics and initial calibration results. In IGARSS '99. 19th International Geoscience and Remote Sensing Symposium, 28 June-2 July 1999, Hamburg, Germany. Proceedings. Vol. 1. Piscataway, NJ, Institute of Electrical and Electronics Engineers, 464-466.

Winebrenner, D. P., L. D. Farmer and I. R. Joughin. 1995. On the response of polarimetric synthetic aperture radar signatures at $24-\mathrm{cm}$ wavelength to sea ice thickness in Arctic leads. Radio Science, 30(2), 373-402. 